\title{
Febrile seizures in children: a condensed update
}

Hong Kong Med J 2019;25:499-500

https://doi.org/10.12809/hkmj198189

To the Editor - We would like to provide a condensed update on febrile seizures in children.

Febrile seizures are the most common type of provoked seizure in children. The cumulative risk varies in different cohorts but is generally reported from $2 \%$ to $5 \% .{ }^{1}$ The typical febrile seizure is a generalised short seizure provoked by a febrile episode in children aged 6 months to 6 years. The duration is shorter than 15 minutes and usually does not recur in the same episode. There are typically no focal neurological deficits or signs of central nervous system (CNS) infection. ${ }^{2}$ Acute management of convulsive seizures, such as resuscitation and firstaid measures, should follow the local prevailing guideline provided by the Hospital Authority intranet.

Diagnosing typical febrile seizure is clinically important and can follow a simple management pathway (Fig). The chance of CNS infection and longterm neurodevelopmental sequelae is low. There is no significant difference in academic performance and behaviour at age 10 years for children who had a typical or atypical febrile seizure compared with control. ${ }^{3}$ Further tests are necessary for fever rather than the seizures themselves.

Lumbar puncture to exclude CNS infection is unnecessary even in infants aged $<1$ year with simple febrile seizures. It should be considered in complex febrile seizures, prolonged seizures, or presence of red flag signs such as meningism, bulging fontanelle, or focal neurological deficit.

Urgent neuroimaging, such as computer tomography or magnetic resonance imaging, should be considered only in prolonged seizures, presence of focal neurological deficits, or prolonged encephalopathic state.

Electroencephalography is of limited value even in complex febrile seizures. It might be considered in focal seizures, presence of focal deficit, persistent alteration of consciousness after seizure (to rule out subclinical or electrographic status epilepticus), or developmental delay.

Counselling is the most important aspect in managing febrile seizures:

1. Prognosis: excellent prognosis for typical febrile seizure; no significant increase in future epilepsy or other neurodevelopmental sequelae for typical febrile seizure even if recurrent; higher risk of sequelae in the presence of atypical features. ${ }^{4}$
2. Recurrence risk: on average $30 \%$ before 6 years old, higher if first attack at a younger age or in the presence of atypical features.

3. Antipyretics are not recommended for febrile seizure prophylaxis.

4. Long-term or intermittent anticonvulsants are useful to reduce recurrence but in general not recommended because of their side-effects.

5. Rescue medication, eg, rectal diazepam should be considered for prolonged febrile seizure, febrile status epilepticus, or patients with limited medical access.

6. Intermittent oral benzodiazepines could be considered for recurrent febrile seizures which are likely predictable, ie, seizures occurred after detection of fever.

7. Caregivers should be taught to manage acute seizures.

\section{Author contributions}

All authors have made substantial contributions to the concept of this study, acquisition and analysis of data, drafting of the article, and critical revision for important intellectual content. All authors had full access to the data, contributed to the study, approved the final version for publication, and take responsibility for its accuracy and integrity.

\section{Conflicts of interest}

All authors have disclosed no conflicts of interest.

\section{Funding/support}

This letter received no specific grant from any funding agency in the public, commercial, or not-for-profit sectors.

\footnotetext{
${ }^{1}$ MM Yau *, FHKAM (Paediatrics)

${ }^{2}$ KL Hon, MD, FAAP

${ }^{3}$ CF Cheng, FHKAM (Paediatrics)
}

\section{Department of Paediatrics and Adolescent Medicine, Tseung Kwan O Hospital, Tseung Kwan O, Hong Kong \\ Department of Paediatrics and Adolescent Medicine, Hong Kong \\ Children's Hospital, Kowloon Bay, Hong Kong \\ 3 Private Practice}

* Corresponding author: yaumanmut@gmail.com

\section{References}

1. Chan KK, Cherk SW, Chan CH, Ng DK, Ho JC. A retrospective review of first febrile convulsion and its risk factors for recurrence in Hong Kong children. HK J Paediatr 2007;12:181-7. 


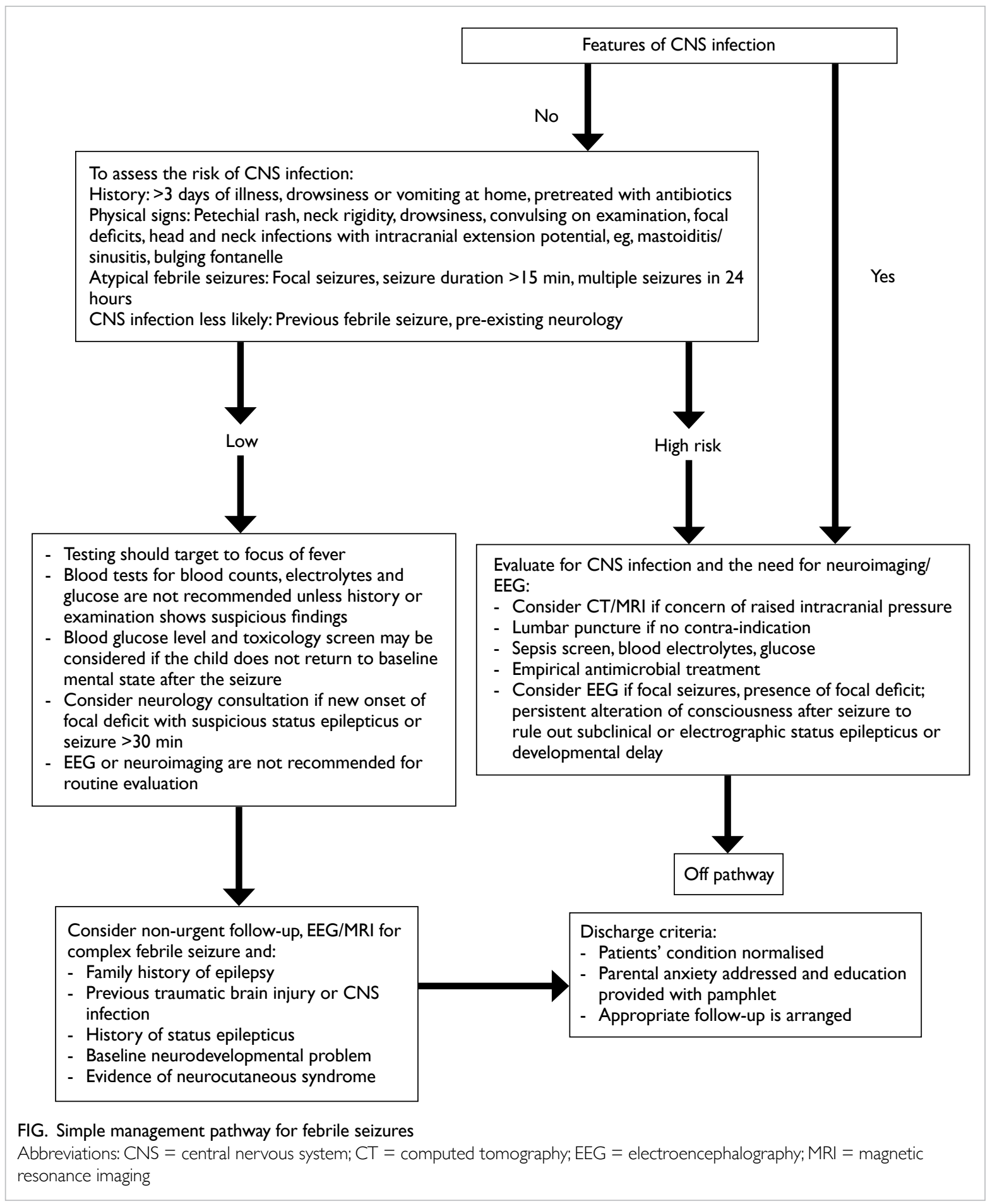

2. Wong V, Ho MH, Rosman NP, et al. Clinical guideline on management of febrile convulsion. HK J Paediatr 2002;7:143-51.

3. Verity CM, Greenwood R, Golding J. Long-term intellectual and behavioral outcomes of children with febrile convulsions. N Engl J Med 1998;338:1723-8.

4. Leung $\mathrm{AK}$, Hon KL, Leung TN. Febrile seizures: an overview. Drugs Context 2018;7:212536. 